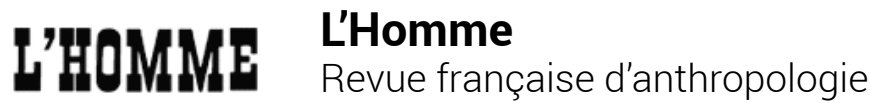

156 | octobre-décembre 2000

Intellectuels en diaspora et théories nomades

\section{Martin de la Soudière, Au bonheur des saisons. Voyage au pays de la météo}

Paris, Bernard Grasset, 1999, 379 p., bibl. raisonnée, index

\section{Bernard Paillard}

\section{(2) OpenEdition}

\section{Journals}

Édition électronique

URL : http://journals.openedition.org/lhomme/2781

DOI : 10.4000/lhomme.2781

ISSN : 1953-8103

Éditeur

Éditions de l'EHESS

Édition imprimée

Date de publication : 1 janvier 2000

Pagination : 313-314

ISBN : 2-7132-1348-7

ISSN : 0439-4216

Référence électronique

Bernard Paillard, « Martin de la Soudière, Au bonheur des saisons. Voyage au pays de la météo »,

L'Homme [En ligne], 156 | octobre-décembre 2000, mis en ligne le 29 novembre 2006, consulté le 24 septembre 2020. URL : http://journals.openedition.org//homme/2781 ; DOI : https://doi.org/10.4000/ Ihomme.2781

Ce document a été généré automatiquement le 24 septembre 2020

(C) École des hautes études en sciences sociales 


\section{Martin de la Soudière, Au bonheur des saisons. Voyage au pays de la météo}

Paris, Bernard Grasset, 1999, 379 p., bibl. raisonnée, index

Bernard Paillard

1 QUE LES jansénistes de la sociologie passent leur chemin : ce livre n'est pas pour eux. Ils n'y trouveront pas leurs repères familiers. Ici, pas d'analyse en termes de catégories sociales, de niveau socioculturel, d'âge, de sexe. Encore moins de statistiques, ni même de grandes envolées théoriques. Car, en paraphrasant l'auteur, on pourrait dire que cet essai, fait de nuances et de variations, relève plus de l'évocation que de l'analyse. C'est sans doute parce que Martin de la Soudière a voulu adapter son écriture à son objet, un objet qui défie la description tant il est en permanent changement : la météo.

2 En effet, «le temps qu'il fait » affecte nos manières de sentir, de dire et de faire. Mais c'est d'une façon versatile comme un nuage, insistante comme une pluie, éphémère comme une belle « embellie ». Et il faut multiplier les capteurs sociologiques pour saisir toutes les « météosensibilités ", tant elles sont diversifiées.

Depuis son ouvrage sur l'hiver ${ }^{1}$, l'auteur a traversé les autres saisons, tenant pendant dix années son "journal météorologique", interrogeant sa propre sensibilité et les humeurs que lui inspiraient le vent, la pluie, la température, le soleil, la neige, le brouillard, tous ces météores qui font l'atmosphère des jours. Il a promené sa curiosité dans les bistrots, sur les marchés, chez les commerçants et a noté ce qu'on disait. Peu d'interviews donc, mais des rencontres plus ordinaires et fugaces, des côtoiements, des «observations de trottoir et de terroir », comme il dit. Il a voyagé en littérature. Il a visité les peintres. Et, scrupuleusement, il a suivi cette météo officielle que nous livrent quotidiennement les médias.

Voilà des matériaux peu coutumiers pour ceux qui, dans leur volonté d'objectivité, ne savent que se mouler dans le cadre strict d'enquêtes standard. C'est pourtant ces chatoiements subtils qu'a choisis Martin de la Soudière comme compagnons de voyage. Vérifiant combien les sensibilités à la météo étaient subjectives et contradictoires, il s'est lui-même positionné, ne refusant pas de donner aux lecteurs ses propres perceptions, impressions, sensations, émotions, appréhensions. Comme s'il voulait en 
permanence non se donner en référence, mais rappeler que l'ethnologue n'est pas un être froid et insensible, étranger au phénomène qu'il étudie. Il doit être un observateur s'observant lui-même. Et Martin de la Soudière n'est pas dupe : s'il parle beaucoup de la pluie, c'est parce qu'il l'aime et qu'il veut quelque peu réhabiliter ce temps haï.

C'est pourquoi son livre est construit de telle sorte que réflexions socioethnographiques et réactions personnelles se côtoient, s'alternent, se renvoient, se contredisent.

6 On l'aura compris, $A u$ bonheur des saisons n'est pas un livre académique. S'il est découpé en chapitres, aucun ne traite d'un seul aspect. On ne peut épuiser ces météores lunatiques. Comme en régime de traîne, on peut parler d'abord de la pluie pour, dans une éclaircie, revenir au soleil. Si l'on traite du brouillard, insensiblement on retrouvera le verglas. Et, toujours, on rejoindra l'auteur et son carnet de route. Tiens, le voici à Guéret. Mais il parle aussi de Paris parce qu'un appel téléphonique lui dit que là aussi, c'est la canicule ou, au contraire, qu'il pleut. Et pourquoi pas faire un tour par le Kenya ou l'île Maurice! Une telle manière peut déconcerter. On n'a pas l'habitude de se laisser ainsi ballotter au gré des nuances. Dans son souci de coller à une réalité si changeante, Martin de la Soudière a un besoin quasi obsessionnel de la précision. On saura que c'est tel ami qui lui a donné tel renseignement, que telle observation a été faite en tel lieu et à telle heure. Et il entend rendre justice à ceux qui, peut-être sans le savoir, l'ont accompagné sur sa route.

On ne peut que louer un tel positionnement de chercheur. Même si, par moments, un trop plein de sincérité personnelle casse le rythme de la lecture. Et l'on peut se perdre dans des allers et retours. Comme on peut se dire que la fusion de deux styles d'exposés (le journal météorologique et la réflexion savante) n'est pas tout à fait accomplie. Pour autant, cette démarche personnelle est à saluer. Il faut oser se présenter ainsi en tentant de marier l'objectif et le subjectif, l'individuel et le collectif. Les sciences humaines ont besoin d'auteurs qui savent être personnels et qui n'assènent pas au lecteur des vérités péremptoires. Elles ont besoin d'ouvrages qui incitent à réfléchir par soi-même.

8 C'est à une lecture impressionniste, avec des touches de pointillisme, que nous invite Martin de la Soudière. Et sa palette, d'une étonnante richesse, associe les couleurs par fondus enchaînés. C'est pourquoi chaque lecteur grappillera au fil du livre, plus qu'il ne trouvera un menu prêt à penser. Par exemple, dans le chapitre III, intitulé «Beaux jours », il pourra s'interroger sur la façon dont il perçoit l'arrivée du printemps, comme il découvrira que si la météo est pleine de clichés, elle relève également d'une sorte de morale intrinsèque. Ne craint-on pas de payer un trop plein de "beau temps ", comme si un comptable avait « des degrés de température à distribuer et à répartir tout au long de l'année, des quotas à respecter, une parité, un équilibre à maintenir entre froid et chaleur, humidité et sécheresse » (p. 74). Au fil des chapitres, il apprendra qu'on vit la météo sur plusieurs registres. Il y a ces modes majeurs, ces temps qui marquent par leur excès de froid, de chaleur, de pluie, et qui parfois provoquent des catastrophes. Ils semblent scander le temps qui passe, même si (chap. XI, « Du temps du ciel au temps qui passe ») cette mémoire météorologique n'est guère infaillible. De même, il saura que la complainte du temps perdu, ce fameux «il n'y a plus de saisons", ne date pas des fusées ni de la bombe atomique. Cette nostalgie d'un âge révolu faisait déjà se lamenter les Grecs. Il se reconnaîtra sans doute dans l'ambiguïté largement partagée qu'on ressent vis-à-vis du brouillard (chap. XIV : «Brouillard journalier »). Vecteur de peur, 
largement utilisé au cinéma, le brouillard peut se rendre bienfaiteur. En fait, l'auteur nous invite à nous demander pourquoi nous passons tant de temps à parler d'une chose aussi insignifiante que le temps. Le plus souvent pour nous en plaindre. Mais aussi, fonction phatique du langage, pour engager ou maintenir le contact avec autrui. Et, dans l'esprit moderne du temps, la météo semble faire partie d'une exigence de qualité de la vie. L'intérêt pour l'atmosphère fait partie de l'air ambiant d'un temps qui a besoin de confort, de protection et de sécurité. Sans doute, là est la mutation qui affecte notre époque. L'obsession pour le ciel ressortit à un besoin psychologique des sociétés urbaines.

\section{NOTES}

1. Martin de la Soudière, L'hiver, à la recherche d'une morte saison, Lyon, La Manufacture, 1987.

\section{AUTEUR}

\section{BERNARD PAILLARD}

EHESS, Centre d'études transdisciplinaires. Sociologie, anthropologie, histoire (CETSAH), Paris. 